\title{
Bioanalysis
}

\section{Self-sampling and quantitative analysis of DBS: can it shift the balance in over-burdened healthcare systems?}

\begin{abstract}
"...the self sampling DBS approach may provide healthcare systems an innovative way of providing evidence-based treatment thereby shifting the balance of healthcare provision from hospitals to the community."
\end{abstract}

Keywords: DBS • LC-HRMS • LC-MS/MS • quantitation • self-collection • self-sampling

\section{Background}

Much is made of the ease of use of DBS in terms of general sample collection and the more widespread utility of the bioanalytical assay resulting from simpler transport requirements. In principal a sample can be collected in locations as diverse as a patient's home or a clinic in sub-Saharan Africa and then sent by post to the laboratory for analysis. The DBS sample can be transported without refrigerants and relatively inexpensively [1]. Not surprisingly DBS sample collection has been used for disease surveillance in developing countries where transport may present problems [2]. The ease of sample collection from a simple fingerprick has made the collection of DBS samples the preferred method amongst prison populations for the monitoring of Hepatitis C [3]. This approach has recently been extended, as a walk-in service, to members of the general public where the uncontrolled use of needles can produce health problems [4].

Perhaps the ultimate use would be to provide an individual with the capability to collect his/her own DBS samples, for analysis, which eliminates the phlebotomy appointment and facilitates access to care for a wider proportion of the community.

Whilst the concept of a 'self-sampled DBS (ssDBS)' is appealing, an individual may find the conventional DBS card approach awkward as highlighted later in this editorial. In parallel to this, there are important considerations if quantitative data are required from the analysis of the DBS sample. In this editorial, we look at what is needed to pro- vide a self-collection DBS protocol capable of producing quantitative data and conclude with results from our investigations using ssDBS samples to monitor therapeutic drug levels in volunteers taking cardiovascular medications.

\section{Drivers for developing a self-sampled DBS capability}

The principal reasons for wanting a ssDBS sample collection capability can be summarized as follows.

Increased privacy of sample collection The test materials and the results can be freely and confidentially available to patients. This approach provides a means for embarrassed, unsure or frightened members of the community to obtain diagnostic information which previously they would not have sought. Principal areas of concern are sexually transmitted infections (STI), HIV and Hepatitis B and C [5].

Increased accessibility and/or usability of sample collection to potential

patients

HIV and malaria sample collection kits can be delivered easily to remote locations in less well-developed countries providing patient accessibility to healthcare. The ease of DBS sample collection means that this is the preferred method for use amongst needle sharing groups, especially in penal environments, since no needles are needed for sample collection (for example for HIV, Hepatitits B and C patients).

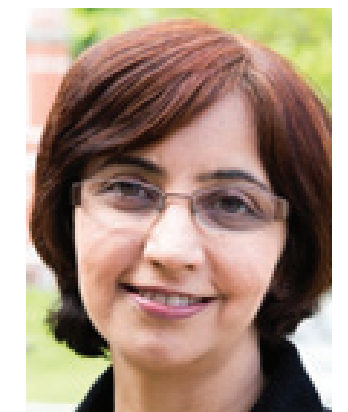

Sangeeta Tanna

Author for correspondence: Leicester School of Pharmacy, Faculty of Health \& Life Sciences, De Montfort University, The Gateway, Leicester, LE1 9BH, UK

Tel.: +44 (0)116 2078274

stanna@dmu.ac.uk

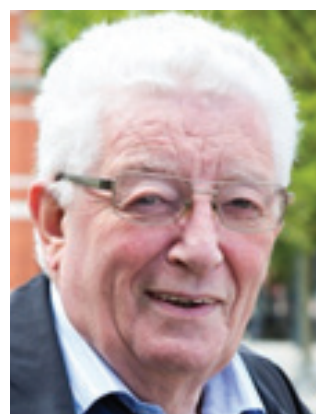

Graham Lawson

Leicester School of Pharmacy, Faculty of Health \& Life Sciences, De Montfort University, The Gateway, Leicester, LE1 9BH, UK 
Ease of sample collection \& transport

A ssDBS capability would remove the need to attend a clinic to provide therapeutic drug level blood samples to aid individual medication. For such applications the collected blood spots are dried and returned by prepaid post for quantitative analysis [6,7].

\section{Reduce the overall costs to the healthcare provider over the long term}

The cost savings associated with the sample transport of conventional DBS sampling are well documented. Little information is, however, available about the savings associated with the early treatment of groups of patients that would become a future burden on the healthcare system. This saving derives from casting the sampling net wider to cover a greater percentage of potential patients [5].

The primary advantages of DBS sample collection, based on information from Yang [8], are summarized below:

- Simplified sample collection, storage and transport;

- Sample stability allowing shipment at ambient temperature to centralized laboratories;

- Minimal sample volume for testing;
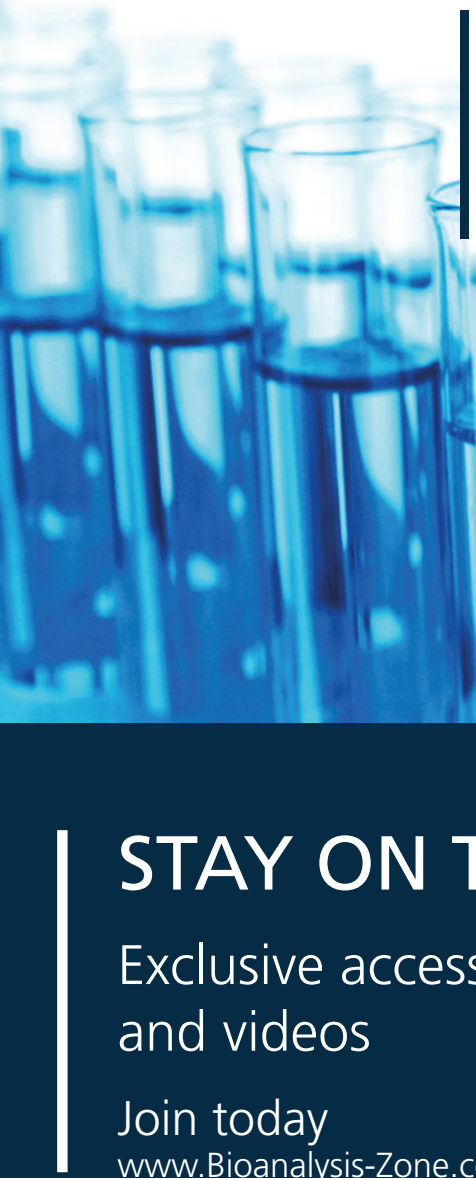

BIOANALYSIS - ZONE

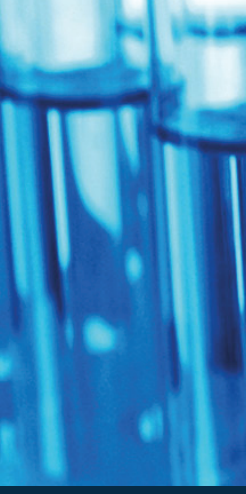

\section{STAY ON TOP}

\section{Exclusive access to news, articles and videos}

Join today

www.Bioanalysis-Zone.com
- Suitability for multiplexed platforms to reduce costs for the health service provider;

- Reduction of blood-borne pathogen transmission through accidental needle sticks as fingerprick sampling is utilized.

These advantages tacitly assume a second person to collect the sample but for 'self-collection' samples a conventional DBS card can present some disadvantages or difficulties:

- Difficult to manipulate card, lancet and maintain pricked finger steady: a task which is all too easy with two people to collect the sample;

- Suitable drying conditions are not always readily available;

- Ensuring the correct amount of blood is sampled and collected in the right place on the card.

These difficulties can be overcome either by adequate training of the patient/volunteer prior to attempting DBS sample collection or by modifying the mode of sample collection to eliminate these difficulties.

\section{New forms of matrix sampling devices}

Cellulose cards represent the majority of the sampling matrices used for DBS. Two novel systems have been developed to make sample self collection easier. The Hemaspot ${ }^{\mathrm{TM}}$ (Spot On Sciences, USA) sampling device [9] is a cartridge containing an absorbent paper and a desiccant covered with an application surface with a small opening to allow entry for two drops of blood. The cartridge is closed and is immediately ready for transport. The Mitra ${ }^{\mathrm{TM}}$ (Phenomenex, USA) microsampling device [10] is based on volumetric absorptive microsampling and collects a consistent volume of blood across a range of hematocrit levels and sampling conditions. Mitra allows easy direct sampling from a subject, requires drying followed by extraction with no punching required. Optimization of the extraction conditions may be required to eliminate hematocrit effects.

\section{Qualitative applications for the ssDBS methodology}

There has been surprisingly little in the published literature concerning ssDBS on cards or other dried matrix sampling devices. The use of DBS cards to confirm disease states is well documented for HIV, malaria and perhaps less so for Hepatitis $B$ and Hepatitis $C$ and the qualitative nature of these determinations makes such applications amenable to ssDBS sampling because spot quality is less demanding. Self-sample collection pro- 
vides a mechanism to provide healthcare to previously unreachable sections of the community; needle sharers, people embarrassed by their actions or conditions or those in remote locations not easily contactable. Such people are found worldwide and not just limited to less well-developed countries.

\section{"The estimated savings to the National Health Service from the early diagnosis of a single HIV case could save $\mathrm{f} 1.2$ million simply by preventing onward transmission and fur- ther cases."}

In the UK, a free ssDBS service is offered under the National Health Service (NHS) 'R U Clear' program [11] for the diagnosis of STIs, initially HIV and syphilis. Test material is supplied following telephone, postal or on-line requests and confidential results are returned by post or text within a few working days. The estimated savings to the NHS from the early diagnosis of a single HIV case could save $£ 1.2$ million simply by preventing onward transmission and further cases. This service is currently under trial in the Manchester and Warrington area of the UK. A similar free and confidential testing service is provided by the Glasgow and Clyde Scots NHS [4] for the diagnoses of cases Hepatitis $\mathrm{C}$ among needle users. Test kits are free and results are supplied by text or post. In the UK, the private supplier Better2Know offers a range of tests, some using the ssDBS approach, to screen for STIs [12]. The value of the ssDBS technique was demonstrated in a study of HIV prevalence and sexual risk behavior amongst men who had sex with men in Paris, France [13]. Almost $18 \%$ of the participants were HIV positive and in this group around $20 \%$ were unaware of their status. Of the total group $21 \%$ were unaware if they were still HIV negative or had never been tested despite their at-risk behavior. This study provided the first estimate of HIV seroprevalence among men who had sex with men in Paris which underlines the value of such studies.

\section{Quantitative ssDBS applications}

Obtaining successful results from a self-sampling exercise, using a conventional DBS card, requires the collection of uniform spots of an appropriate size in the correct positions on the card. Work at De Montfort University (DMU) [6,7] on the viability of ssDBS collection paralleled that reported by Li et al. [14] with the preparation of detailed instruction sheets for the study subjects on how to collect their own DBS samples. In both investigations one-to-one training and practice in sample collection was provided for the volunteers. In both, similar sampling kits were provided to the volunteers. Li et al. focused on the determination of the levels of a drug new to the volunteers whereas at DMU the objective was the quantitative determination of selected cardiovascular disease drugs the volunteers confirmed they were prescribed. Volunteers at DMU were asked to collect repeat samples at different times after taking their normal dose. A fully validated analysis of DBS samples for the selected cardiovascular disease drugs has been reported elsewhere and will not be repeated here. The primary concern of both investigations was the ability of the subjects to collect acceptable blood spots on their own away from the clinic and so all returned cards were checked to ensure the quality of the collected spots.

One of the volunteers at DMU admitted an inability to self sample despite having collected many newborn screening 'heel prick' samples. Around 19\% of the sample cards at DMU versus $17 \%$ reported by Li et al., were rejected for unacceptably sized, misshapen or other problems with the blood spots. In both investigations the accepted blood spots gave data consistent with expectations. Whether the agreement in these two investigations on the level of acceptable ssDBS cards is coincidental cannot be determined but indications are that ssDBS sample collection is a viable protocol.

\section{Conclusion}

With a rapidly increasing elderly population in which multimorbidities are common and for whom attendance at a phlebotomy clinic adds a further layer of complexity, the ssDBS approach may provide healthcare systems an innovative way of providing evidence-based treatment thereby shifting the balance of healthcare provision from hospitals to the community. The vision is for a paradigm shift in the investigations of various clinical conditions involving blood sampling away from hospital-centered activity toward patient-focused sampling in order to widen access to healthcare. This can be achieved by reducing the pressure on the acute care services, making sample collection easier for the patients and by reaching sections of the population either too intimidated or too embarrassed to seek help.

\section{Financial \& competing interests disclosure}

The authors have no relevant affiliations or financial involvement with any organization or entity with a financial interest in or financial conflict with the subject matter or materials discussed in the manuscript. This includes employment, consultancies, honoraria, stock ownership or options, expert testimony, grants or patents received or pending, or royalties.

No writing assistance was utilized in the production of this manuscript. 


\section{References}

1 Mei J. Dried blood spot sample collection, storage and transportation. In: Dried Blood Spots. Applications and Techniques. Li W, Lee MS (Eds). Wiley, NJ, USA, 21-31 (2014).

2 Basavaraja SV, Pitman JP. Dried blood spots for use in HIVrelated epidemiological studies in resource-limited settings. In: Dried Blood Spots. Applications and Techniques. Li W, Lee MS (Eds). Wiley, NJ, USA, 76-94 (2014).

3 Dodd J, Noble J, Jenkins L et al. Increasing hepatitis B and C testing in the prison setting. The Hepatitis $\mathrm{C}$ Trust. www.hepctrust.org.uk

4 Greater Glasgow Hepatitis Outreach Service Evaluation Report.

www.hepcnet.scot.nhs.uk

5 Tait JM, Stephens BP, McIntyre PG et al. Dry blood spot testing for Hepatitis $\mathrm{C}$ in people who injected drugs: reaching the populations other tests cannot reach. Frontline Gastroenterol. 4(4), 255-262 (2013).

6 Lawson G, Cocks E, Tanna S. Bisoprolol, ramipril and simvastatin determination in dried blood spot samples using LC-HRMS for assessing medication adherence. J. Pharm. Biomed. Anal. 81-82, 99-107 (2013).

7 Tanna S, Lawson G. Dried blood spot analysis to assess medication adherence and to inform personalization of treatment. Bioanalysis 6(21), 2825-2838 (2014).
8 Yang C. Dried blood spot specimens for polymerase chain reaction in molecular diagnostics and public health surveillance. In: Dried Blood Spots. Applications and Techniques. Li W, Lee MS (Eds). Wiley, NJ, USA, 32-33 (2014).

9 Spot On Sciences. www.spotonsciences.com/hemaspot

10 Kushon S, Bischofberger A, Carpenter A et al. A novel dried matrix microsampling device that eliminates the volume based haematocrit bias associated with DBS sub-punch workflows.

http://blob.phenomenex.com

11 The sexual health network. NHS Greater Manchester. www.ruclear.co.uk

12 Better 2 Know - Home Testing STD/STI Testing Kits. http://home.better2know.co.uk/home-testing-kits

13 Velter A, Barin F, Bouyssou A et al. HIV prevalence and sexual risk behaviors associated with awareness of HIV status among men who have sex with men in Paris, France. Aids Behav. 17(4), 1266-1278 (2013).

14 Li W, Doherty J, Moench P et al. LC-MS/MS bioanalysis of loratadine (Claritin) in dried blood spot (DBS) samples collected by subjects in a clinical research study. J. Chromatogr. B Analyt. Technol. Biomed. Life Sci. 983, 117-124 (2015). 